\title{
Mean ergodic theorem for semigroups of linear operators in multi-Banach spaces
}

\author{
Hassan Morghi Kenari ${ }^{1}$, Reza Saadati ${ }^{2}$, Mahdi Azhini' and Yeol Je Cho ${ }^{3 *}$
}

\author{
"Correspondence: yjcho@gnu.ac.kr \\ ${ }^{3}$ Department of Mathematics \\ Education and the RINS, \\ Gyeongsang National University, \\ Jinju, 660-701, Korea \\ Full list of author information is \\ available at the end of the article
}

\begin{abstract}
In this paper, by using Rodé's method, we extend Yosida's theorem to semigroups of linear operators in multi-Banach spaces.

MSC: Primary 39A10; 39B72; secondary 47H10; 46B03
\end{abstract}

Keywords: ergodic theorem; semigroups; multi-Banach space

\section{Introduction}

In 1938, Yosida [1] proved the following mean ergodic theorem for linear operators: Let $E$ be a real Banach space and $T_{j}(j=1,2, \ldots)$ be linear operators of $E$ into itself such that there exists a constant $C$ with $\left\|\left(T_{1}^{n}, \ldots, T_{j}^{n}\right)\right\| \leq C$ for $n=1,2,3, \ldots$, and $T_{j}$ is weakly completely continuous, i.e., $T_{j}$ maps the closed unite ball of $E$ into a weakly compact subset of $E$. Then the Cesaro means

$$
S_{n, j} x=\frac{1}{n} \sum_{k=1}^{n} T_{j}^{k} x
$$

converges strongly as $n \rightarrow+\infty$ to a fixed point of $T_{j}$ for each $x \in E$.

On the other hand, in 1975, Baillon [2] proved the following nonlinear ergodic theorem. Let $X$ be a Banach space and $C$ be a closed convex subset of $X$. The mappings $T_{j}: C \rightarrow C$ $(j=1,2, \ldots)$ are called nonexpansive on $C$ if

$$
\left\|T_{j} x-T_{j} y\right\| \leq\|x-y\| \quad \forall x, y \in C .
$$

Let $F\left(T_{j}\right)$ be the set of fixed points of $T_{j}$. If $X$ is strictly convex, $F\left(T_{j}\right)$ is closed and convex. In [2], Baillon proved the first nonlinear ergodic theorem such that if $X$ is a real Hilbert space and $F\left(T_{j}\right) \neq \emptyset$, then for each $x \in C$, the sequence $\left\{S_{n, j} x\right\}$ defined by

$$
S_{n, j} x=\left(\frac{1}{n}\right)\left(x+T_{j} x+\cdots+T_{j}^{n-1} x\right)
$$

converges weakly to a fixed point of $T_{j}$. It was also shown by Pazy [3] that if $X$ is a real Hilbert space and $S_{n, j} x$ converges weakly to $y \in C$, then $y \in F\left(T_{j}\right)$.

Recently, Rodé [4] and Takahashi [5] tried to extend this nonlinear ergodic theorem to semigroup, generalizing the Cesaro means on $\mathbf{N}=\{1,2, \ldots\}$, such that the corresponding

\section{Springer}

(2014 Kenari et al.; licensee Springer. This is an Open Access article distributed under the terms of the Creative Commons Attribution License (http://creativecommons.org/licenses/by/2.0), which permits unrestricted use, distribution, and reproduction in any medium, provided the original work is properly cited. 
sequence of mappings converges to a projection onto the set of common fixed points. In this paper, by using Rodé's method, we extend Yosida's theorem to semigroups of linear operators in multi-Banach spaces. The proofs employ the methods of Yosida [1], Rodé [4], Greenleaf [6] and Takahashi [7, 8]. Our paper is motivated from ideas in [9].

\section{Multi-Banach spaces}

The notion of multi-normed space was introduced by Dales and Polyakov in [10]. This concept is somewhat similar to operator sequence space and has some connections with operator spaces and Banach lattices. Motivations for the study of multi-normed spaces and many examples are given in [10-16].

Let $(E,\|\cdot\|)$ be a complex normed space, and let $k \in \mathbf{N}$. We denote by $E^{k}$ the linear space $E \oplus \cdots \oplus E$ consisting of $k$-tuples $\left(x_{1}, \ldots, x_{k}\right)$, where $x_{1}, \ldots, x_{k} \in E$. The linear operations on $E^{k}$ are defined coordinate-wise. The zero element of either $E$ or $E^{k}$ is denoted by 0 . We denote by $\mathbf{N}_{k}$ the set $\{1,2, \ldots, k\}$ and by $\Sigma_{k}$ the group of permutations on $k$ symbols.

Definition 2.1 Let $E$ be a linear space, and take $k \in \mathbf{N}$. For $\sigma \in \Sigma_{k}$, define

$$
A_{\sigma}(x)=\left(x_{\sigma(1)}, \ldots, x_{\sigma(k)}\right), \quad x=\left(x_{1}, \ldots, x_{k}\right) \in E^{k} .
$$

For $\alpha=\left(\alpha_{i}\right) \in \mathbf{C}^{k}$, define

$$
M_{\alpha}(x)=\left(\alpha_{i} x_{i}\right), \quad x=\left(x_{1}, \ldots, x_{k}\right) \in E^{k} .
$$

Definition 2.2 Let $(E,\|\cdot\|)$ be complex (respectively, real) normed space, and take $n \in \mathbf{N}$. A multi-norm of level $n$ on $\left\{E^{k}: k \in \mathbf{N}_{n}\right\}$ is a sequence $\left(\|\cdot\|_{k}: k \in \mathbf{N}_{n}\right)$ such that $\|\cdot\|$ is a norm on $E^{k}$ for each $k \in \mathbf{N}_{n}$, such that $\|x\|_{1}=\|x\|$ for each $x \in E$ (so that $\|\cdot\|_{1}$ is the initial norm), and such that the following axioms (A1)-(A4) are satisfied for each $k \in \mathbf{N}_{n}$ with $k \geq 2$ :

(A1) for each $\sigma \in \Sigma_{k}$ and $x \in E^{k}$, we have

$$
\left\|A_{\sigma}(x)\right\|_{k}=\|x\|_{k}
$$

(A2) for each $\alpha_{1}, \ldots, \alpha_{k} \in \mathbf{C}$ (respectively, each $\alpha_{1}, \ldots, \alpha_{k} \in \mathbf{R}$ ) and $x \in E^{k}$, we have

$$
\left\|M_{\alpha}(x)\right\|_{k} \leq\left(\max _{i \in \mathbf{N}_{k}}\left|\alpha_{i}\right|\right)\|x\|_{k}
$$

(A3) for each $x_{1}, \ldots, x_{k-1}$, we have

$$
\left\|\left(x_{1}, \ldots, x_{k-1}, 0\right)\right\|_{k}=\left\|\left(x_{1}, \ldots, x_{k-1}\right)\right\|_{k-1} ;
$$

(A4) for each $x_{1}, \ldots, x_{k-1} \in E$

$$
\left\|\left(x_{1}, \ldots, x_{k-2}, x_{k-1}, x_{k-1}\right)\right\|_{k}=\left\|\left(x_{1}, \ldots, x_{k-1}, x_{k-1}\right)\right\|_{k-1} .
$$

In this case, $\left(\left(E^{k},\|\cdot\|_{k}\right): k \in \mathbf{N}_{n}\right)$ is a multi-normed space of level $n$. 
A multi-norm on $\left\{E^{k}: k \in \mathbf{N}\right\}$ is a sequence

$$
\left(\|\cdot\|_{k}\right)=\left(\|\cdot\|_{k}: k \in \mathbf{N}\right)
$$

such that $\left(\|\cdot\|_{k}: k \in \mathbf{N}_{n}\right)$ is a multi-norm of level $n$ for each $n \in \mathbf{N}$. In this case, $\left(\left(E^{n},\|\cdot\|_{n}\right)\right.$ : $n \in \mathbf{N}$ ) is a multi-normed space.

Lemma 2.3 [12] Suppose that $\left(\left(E^{k},\|\cdot\|_{k}\right): k \in \mathbf{N}\right)$ is a multi-normed space, and take $k \in$ $\mathbf{N}_{n}$. Then

(a) $\|(x, \ldots, x)\|_{k}=\|x\|(x \in E)$;

(b) $\max _{i \in \mathbf{N}_{k}}\left\|x_{i}\right\| \leq\left\|\left(x_{1}, \ldots, x_{k}\right)\right\|_{k} \leq \sum_{i=1}^{k}\left\|x_{i}\right\| \leq k \max _{i \in \mathbf{N}_{k}}\left\|x_{i}\right\|\left(x_{1}, \ldots, x_{k} \in E\right)$.

It follows from (b) that, if $(E,\|\cdot\|)$ is a Banach space, then $\left(E^{k},\|\cdot\|_{k}\right)$ is a Banach space for each $k \in \mathbf{N}$; in this case $\left(\left(E^{k},\|\cdot\|_{k}\right): k \in \mathbf{N}\right)$ is a multi-Banach space.

Now we state two important examples of multi-norms for an arbitrary normed space $E$; cf. [10].

Example 2.4 The sequence $\left(\|\cdot\|_{k}: k \in \mathbf{N}\right)$ on $\left\{E^{k}: k \in \mathbf{N}\right\}$ defined by

$$
\left\|\left(x_{1}, \ldots, x_{k}\right)\right\|_{k}:=\max _{i \in \mathbf{N}_{k}}\left\|x_{i}\right\| \quad\left(x_{1}, \ldots, x_{k} \in E\right)
$$

is a multi-norm called the minimum multi-norm. The terminology 'minimum' is justified by property (b).

Example 2.5 Let $\left\{\left(\|\cdot\|_{k}^{\alpha}: k \in \mathbf{N}\right): \alpha \in A\right\}$ be the (non-empty) family of all multi-norms on $\left\{E^{k}: k \in \mathbf{N}\right\}$. For $k \in \mathbf{N}$, set

$$
\left\|\left(x_{1}, \ldots, x_{k}\right)\right\|_{k}:=\sup _{\alpha \in A}\left\|\left(x_{1}, \ldots, x_{k}\right)\right\|_{k}^{\alpha} \quad\left(x_{1}, \ldots, x_{k} \in E\right)
$$

Then $\left(\|\cdot\|_{k}: k \in \mathbf{N}\right)$ is a multi-norm on $\left\{E^{k}: k \in \mathbf{N}\right\}$, called the maximum multi-norm.

We need the following observation, which can easily be deduced from the triangle inequality for the norm $\|\cdot\|_{k}$ and the property (b) of multi-norms.

Lemma 2.6 Suppose that $k \in \mathbf{N}$ and $\left(x_{1}, \ldots, x_{k}\right) \in E^{k}$. For each $j \in\{1, \ldots, k\}$, let $\left(x_{n}^{j}\right)_{n=1,2, \ldots}$ be a sequence in $E$ such that $\lim _{n \rightarrow \infty} x_{n}^{j}=x_{j}$. Then for each $\left(y_{1}, \ldots, y_{k}\right) \in E^{k}$ we have

$$
\lim _{n \rightarrow \infty}\left(x_{n}^{1}-y_{1}, \ldots, x_{n}^{k}-y_{k}\right)=\left(x_{1}-y_{1}, \ldots, x_{k}-y_{k}\right)
$$

Definition 2.7 Let $\left(\left(E^{k},\|\cdot\|_{k}\right): k \in \mathbf{N}\right)$ be a multi-normed space. A sequence $\left(x_{n}\right)$ in $E$ is a multi-null sequence if, for each $\varepsilon>0$, there exists $n_{0} \in \mathbf{N}$ such that

$$
\sup _{k \in \mathbf{N}}\left\|\left(x_{n}, \ldots, x_{n+k-1}\right)\right\|_{k}<\varepsilon \quad\left(n \geq n_{0}\right) .
$$

Let $x \in \mathcal{E}$. We say that the sequence $\left(x_{n}\right)$ is multi-convergent to $x \in E$ and write

$$
\lim _{n \rightarrow \infty} x_{n}=x
$$

if $\left(x_{n}-x\right)$ is a multi-null sequence. 


\section{Preliminaries and lemmas}

Let $E$ a real Banach space and let $E^{*}$ be the conjugate space of $E$, that is, the space of all continuous linear functionals on $E$. The value of $x^{*} \in E^{*}$ at $x \in E$ will be denoted by $\left\langle x, x^{*}\right\rangle$. We denote by $\operatorname{co} D$ the convex hull of $D, \overline{c o} D$ the closure of $\operatorname{co} D$.

Let $U$ be a linear continuous operator of $E$ into itself. Then we denote by $U^{*}$ the conjugate operator of $U$.

Assumption (A) Let $\left(E^{j},\|\cdot\|_{j}\right)$ be a multi-Banach space and $\left\{T_{j, t}: t \in G\right\}(j=1,2, \ldots)$ be a family of linear continuous operators of a real Banach space $E_{j}$ into itself such that there exists a real number $C$ with $\left\|\left(T_{1, t}, \ldots, T_{j, t}\right)\right\|_{j} \leq C$ for all $t \in G$ and the weak closure of $\left\{T_{j, t} x: t \in G\right\}$ is weakly compact, for each $x \in E$. The index set $G$ is a topological semigroup such that $T_{j, s t}=T_{j, s} \cdot T_{j, t}$ for all $s, t \in G$ and $T_{j}$ is continuous with respect to the weak operator topology: $\left\langle T_{j, s} x, x^{*}\right\rangle \rightarrow\left\langle T_{j, t} x, x^{*}\right\rangle$ for all $x \in E$ and $x^{*} \in E^{*}$ if $s \rightarrow t$ in $G$.

We denote by $m_{j}(G)$ the Banach space of all bounded continuous real valued functions on the topological semigroup $G$ with the supremum norm. For each $s \in G$ and $f_{j} \in m_{j}(G)$, we define elements $l_{s} f_{j}$ and $r_{s} f_{j}$ in $m_{j}(G)$ given by $l_{s} f_{j}(t)=f_{j}(s t)$ and $r_{s} f_{j}(t)=f_{j}(t s)$ for all $t \in G$. An element $\mu_{j} \in m_{j}(G)^{*}$ (the conjugate space of $m_{j}(G)$ ) is called a mean on $G$ if $\left\|\mu_{j}\right\|=$ $\mu_{j}(1)=1$ moreover, we have $\left\|\left(\mu_{1}, \ldots, \mu_{j}\right)\right\|_{j}=\frac{\sum_{i=1}^{j} \mu_{i}(1)}{j}=1$. A mean $\mu_{j}$ on $G$ is called left (right) invariant if $\mu_{j}\left(l_{s} f_{j}\right)=\mu_{j}\left(f_{j}\right)\left(\mu_{j}\left(r_{s} f_{j}\right)=\mu_{j}\left(f_{j}\right)\right)$ for all $f_{j} \in m_{j}(G)$ and $s \in G$. An invariant mean is a left and right invariant mean. We know that $\mu_{j} \in m_{j}(G)^{*}$ is a mean on $G$ if and only if

$$
\inf \left\{f_{j}(t): t \in G\right\} \leq \mu_{j}\left(f_{j}\right) \leq \sup \left\{f_{j}(t): t \in G\right\}
$$

for every $f_{j} \in m_{j}(G)$; see $[6,17-20]$.

Let $\left\{T_{j, t}: t \in G\right\}$ be a family of linear continuous operators of $E$ into itself satisfying Assumption (A) and $\mu_{j}$ be a mean on G. Fix $x \in E$. Then, for $x^{*} \in E^{*}$, the real valued function $t \rightarrow\left\langle T_{j, t} x, x^{*}\right\rangle$ is in $m_{j}(G)$. Denote by $\mu_{j, t}\left\langle T_{j, t} x, x^{*}\right\rangle$ the value of $\mu_{j}$ at this function. By linearity of $\mu_{j}$ and of $\langle\cdot, \cdot\rangle$, this is linear in $x^{*}$; moreover, since

$$
\begin{aligned}
& \left.\left.\left|\left(\mu_{1, t} \mid T_{1, t} x, x^{*}\right), \ldots, \mu_{j, t}\right| T_{j, t} x, x^{*}\right\rangle\right) \mid \\
& \quad \leq\left\|\left(\mu_{1}, \ldots, \mu_{j}\right)\right\|_{j} \cdot \sup _{t}\left|\left(\left\langle T_{1, t} x, x^{*}\right\rangle, \ldots, \mu_{j, t}\left\langle T_{j, t} x, x^{*}\right\rangle\right)\right| \\
& \quad \leq \sup _{t}\left\|\left(T_{1} x, \ldots, T_{j} x\right)\right\|_{j} \cdot\left\|x^{*}\right\|_{j} \\
& \quad \leq C \cdot\|x\|_{j} \cdot\left\|x^{*}\right\|_{j}
\end{aligned}
$$

it is continuous in $x^{*}$. Hence we find that $\mu_{j, t}\left\langle T_{j, t} x, \cdot\right\rangle$ is an element of $E^{* *}$. So, from weak compactness of $\overline{\operatorname{co}}\left\{T_{j, t} x: t \in G\right\}$ such that $\mu_{j, t}\left\langle T_{j, t} x, x^{*}\right\rangle=\left\langle T_{j, \mu_{j}} x, x^{*}\right\rangle$ for every $x^{*} \in E^{*}$.

Put $K=\overline{\operatorname{co}}\left\{T_{j, t} x: t \in G\right\}$ and suppose that the element $\mu_{j, t}\left\langle T_{j, t} x, \cdot\right\rangle$ is not contained in the $n(K)$, where $n$ is the natural embedding of the Banach space $E$ into its second conjugate space $E^{* *}$. Then, since the convex set $n(K)$ is compact in the weak* topology of $E^{* *}$, there exists an element $y^{*} \in E^{*}$ such that

$$
\mu_{j, t}\left\langle T_{j, t} x, y^{*}\right\rangle<\inf \left\{\left\langle y^{*}, z^{* *}\right\rangle: z^{* *} \in n(k)\right\} .
$$


Hence, we have

$$
\begin{aligned}
\mu_{j, t}\left\langle T_{j, t} x, y^{*}\right\rangle & <\inf \left\{\left\langle y^{*}, z^{* *}\right\rangle: z^{* *} \in n(k)\right\} \\
& \leq \inf \left\{\left\langle T_{j, t} x, y^{*}\right\rangle: t \in G\right\} \\
& \leq \mu_{j, t}\left\langle T_{j, t} x, y^{*}\right\rangle .
\end{aligned}
$$

This is a contradiction. Thus, for a mean $\mu_{j}$ on $G$, we can define a linear continuous operator $T_{j, \mu_{j}}$ of $E$ into itself such that $\left\|\left(T_{1, \mu_{1}}, \ldots, T_{j, \mu_{j}}\right)\right\|_{j} \leq C, T_{j, \mu_{j}} x \in \overline{c o}\left\{T_{j, t} x: t \in G\right\}$ for all $x \in E$, and $\mu_{j, t}\left\langle T_{j, t} x, x^{*}\right\rangle=\left\langle T_{j, \mu_{j}} x, x^{*}\right\rangle$ for all $x \in E$ and $x^{*} \in E^{*}$. We denote by $F_{j}(G)$ the set all common fixed points of the mappings $T_{j, t}, t \in G$.

Lemma 3.1 Assume that a left invariant mean $\mu_{j}$ exists on $G$, then $T_{j, \mu_{j}}(E) \subset F_{j}(G)$. Especially, $F_{j}(G)$ is then not empty.

Proof Let $x \in E$ and $\mu$ be a left invariant mean on $G$. Then since, for $s \in G$ and $x^{*}$,

$$
\begin{aligned}
\left\langle T_{j, s} T_{j, \mu_{j}} x, x^{*}\right\rangle & =\left\langle T_{j, \mu_{j}} x, T_{j, s}^{*} x^{*}\right\rangle \\
& =\mu_{j, t}\left\langle T_{j, t} x, T_{j, s}^{*} x^{*}\right\rangle=\mu_{j, t}\left\langle T_{j, s} T_{j, t} x, x^{*}\right\rangle \\
& =\mu_{j, t}\left\langle T_{j, s t} x, x^{*}\right\rangle=\mu_{j, t}\left\langle T_{j, t} x, x^{*}\right\rangle \\
& =\left\langle T_{j, \mu_{j}} x, x^{*}\right\rangle,
\end{aligned}
$$

we have $T_{j, s} T_{j, \mu_{j}} x=T_{j, \mu_{j}} x$. Hence, $T_{j, \mu_{j}}(E) \subset F_{j}(G)$.

Lemma 3.2 Let $\lambda_{j}$ be an invariantmean on $G$. Then $T_{j, \lambda_{j}} T_{j, s}=T_{j, s} T_{j, \lambda_{j}}=T_{j, \lambda_{j}}$ for each $s \in G$ and $T_{j, \lambda_{j}} T_{j, \mu_{j}}=T_{j, \mu_{j}} T_{j, \lambda_{j}}=T_{j, \lambda_{j}}$ for each mean $\mu_{j}$ on G. Especially, $T_{j, \lambda_{j}}$ is a projection of $E$ onto $F(G)$.

Proof Let $s \in G$. Then, since

$$
\begin{aligned}
\left\langle T_{j, \lambda_{j}} T_{j, s} x, x^{*}\right\rangle & =\lambda_{j, t}\left\langle T_{j, t} T_{j, s} x, x^{*}\right\rangle=\lambda_{j, t}\left\langle T_{j, t s} x, x^{*}\right\rangle \\
& =\lambda_{j, t}\left\langle T_{j, t} x, x^{*}\right\rangle=\left\langle T_{j, \lambda_{j}} x, x^{*}\right\rangle
\end{aligned}
$$

for $x \in E$ and $x^{*} \in E^{*}$, we have $T_{j, \lambda_{j}} T_{j, s}=T_{j, \lambda_{j}}$. It is obvious from Lemma 3.1 that $T_{j, s} T_{j, \lambda_{j}}=$ $T_{j, \lambda_{j}}$ for each $s \in G$. Let $\mu_{j}$ be a mean on $G$. Then, since

$$
\begin{aligned}
\left\langle T_{j, \mu_{j}} T_{j, \lambda_{j}} x, x^{*}\right\rangle & =\mu_{j, t}\left\langle T_{j, t} T_{j, \lambda_{j}} x, x^{*}\right\rangle=\left\langle\mu_{j, t} T_{j, \lambda_{j}} x, x^{*}\right\rangle \\
& =\left\langle T_{j, \lambda_{j}} x, x^{*}\right\rangle
\end{aligned}
$$

and

$$
\begin{aligned}
\left\langle T_{j, \lambda_{j}} T_{j, \mu_{j}} x, x^{*}\right\rangle & =\left\langle T_{j, \mu_{j}} x, T_{j, \lambda_{j}}^{*} x^{*}\right\rangle=\mu_{j, t}\left\langle T_{j, t} x, T_{j, \lambda_{j}}^{*} x^{*}\right\rangle \\
& =\mu_{j, t}\left\langle T_{j, \lambda_{j}} T_{j, t} x, x^{*}\right\rangle=\mu_{j, t}\left\langle T_{j, \lambda_{j}} x, x^{*}\right\rangle \\
& =\left\langle T_{j, \lambda_{j}} x, x^{*}\right\rangle
\end{aligned}
$$


for $x \in E$ and $x^{*} \in E^{*}$, we have $T_{j, \mu_{j}} T_{j, \lambda_{j}}=T_{j, \lambda_{j}} T_{j, \mu_{j}}=T_{j, \lambda_{j}}$. Putting $\mu_{j}=\lambda_{j}$, we have $T_{\lambda_{j}}^{2}=T_{\lambda_{j}}$ and hence $T_{\lambda_{j}}$ is a projection of $E$ onto $F_{j}(G)$.

As direct consequence of Lemma 3.2, we have the following.

Lemma 3.3 Let $\mu_{j}$ and $\lambda_{j}$ be invariant means on G. Then $T_{j, \mu_{j}}=T_{j, \lambda_{j}}$.

Lemma 3.4 Assume that an invariant mean exists on $G$. Then, for each $x \in E$, the set $\overline{\mathrm{co}}\left\{T_{j, t} x: t \in G\right\} \cap F_{j}(G)$ consists of a single point.

Proof Let $x \in E$ and $\mu_{j}$ be an invariant mean on $G$. Then we know that $T_{j, \mu_{j}} x \in F_{j}(G)$ and $T_{j, \mu_{j}} x \in \overline{\mathrm{co}}\left\{T_{j, t} x: t \in G\right\}$. So, we show that $\overline{\operatorname{co}}\left\{T_{j, t} x: t \in G\right\} \cap F_{j}(G)=\left\{T_{j, \mu_{j}} x\right\}$. Let $x_{0} \in \overline{c o}\left\{T_{j, t} x: t \in G\right\} \cap F_{j}(G)$ and $\epsilon>0$. Then, for $x^{*} \in E^{*}$, there exists an element $\sum_{i=1}^{n} \alpha_{i} T_{j, t_{i}} x$ in the set $\operatorname{co}\left\{T_{j, t} x: t \in G\right\}$ such that $\epsilon>C \cdot\left\|x^{*}\right\|_{j} \cdot\left\|\sum_{i=1}^{n} \alpha_{i} T_{j, t_{i}} x-x_{0}\right\|_{j}$. Hence, we have

$$
\begin{aligned}
\epsilon & >C \cdot\left\|x^{*}\right\|_{j} \cdot\left\|\sum_{i=1}^{n} \alpha_{i} T_{j, t_{i}} x-x_{0}\right\|_{j} \\
& \geq \sup _{t}\left\|T_{j, t}\right\|_{j} \cdot\left\|\sum_{i=1}^{n} \alpha_{i} T_{j, t_{i}} x-x_{0}\right\|_{j} \cdot\left\|x^{*}\right\|_{j} \\
& \geq \sup _{t}\left\|\sum_{i=1}^{n} \alpha_{i} T_{j, t} T_{j, t_{i}} x-x_{0}\right\|_{j} \cdot\left\|x^{*}\right\|_{j} \\
& \geq\left|\left\langle\sum_{i=1}^{n} \alpha_{i} T_{j, t} T_{j, t_{i}} x-x_{0}, x^{*}\right\rangle\right| \\
& =\left|\sum_{i=1}^{n} \alpha_{i} \mu_{j, t}\left\langle T_{j, t t_{i}} x-x_{0}, x^{*}\right\rangle\right| \\
& =\left|\mu_{j, t}\left\langle T_{j, t} x-x_{0}, x^{*}\right\rangle\right| \\
& =\left|\left\langle T_{j, \mu_{j}} x-x_{0}, x^{*}\right\rangle\right| .
\end{aligned}
$$

Since $\epsilon$ is arbitrary, we have $\left\langle T_{j, \mu_{j}} x, x^{*}\right\rangle=\left\langle x_{0}, x^{*}\right\rangle$ for every $x^{*} \in E^{*}$ and hence $T_{j, \mu_{j}} x=x_{0}$.

\section{Ergodic theorems}

Now, we can prove mean ergodic theorems for semigroups of linear continuous operators in multi-Banach space.

Theorem 4.1 Let $\left\{T_{j, t}: t \in G\right\}$ be a family of linear continuous operators in a real Banach space $E$ satisfying Assumption (A). If a net $\left\{\mu_{j}^{\alpha}: \alpha \in I\right\}$ of means on $G$ is asymptotically invariant, i.e.,

$$
\mu_{j}^{\alpha}-r_{s}^{*} \mu_{j}^{\alpha} \quad \text { and } \quad \mu_{j}^{\alpha}-l_{s}^{*} \mu_{j}^{\alpha}
$$

converge to 0 in the weak $k^{*}$ topology of $m_{j}(G)^{*}$ for each $s \in G$, then there exists a projection $Q_{j}$ of $E$ on to $F_{j}(G)$ such that $\left\|\left(Q_{1}, \ldots, Q_{j}\right)\right\|_{j} \leq C, T_{j, \mu_{j}^{\alpha}} x$ converges weakly to $Q_{j} x$ for each $x \in E$, 
$Q_{j} T_{j, t}=T_{j, t} Q_{j}=Q_{j}$ for each $t \in G$, and $Q_{j} x \in \overline{c o}\left\{T_{j, t} x: t \in G\right\}$ for each $x \in E$. Furthermore, the projection $Q_{j}$ onto $F_{j}(G)$ is the same for all asymptotically invariant nets.

Proof Let $\mu_{j}$ be a cluster point of net $\left\{\mu_{j}^{\alpha}: \alpha \in I\right\}$ in the weak* topology of $m_{j}(G)^{*}$. Then $\mu_{j}$ is an invariant mean on $G$. Hence, by Lemma 3.2, $T_{j, \mu_{j}}$ is a projection of $E$ onto $F_{j}(G)$ such that $\left\|\left(T_{1, \mu_{1}}, \ldots, T_{j, \mu_{j}}\right)\right\|_{j} \leq C, T_{j, \mu_{j}} T_{j, t}=T_{j, t} T_{j, \mu_{j}}=T_{j, \mu_{j}}$ for each $t \in G$ and $T_{j, \mu_{j}} x \in \overline{\operatorname{co}}\left\{T_{j, t} x: t \in G\right\}$ for each $x \in E$. Setting $Q_{j}=T_{j, \mu_{j}}$, we show that $T_{j, \mu_{j}^{\alpha}} x$ converges weakly to $Q_{j} x$ for each $x \in E$. Since $T_{j, \mu_{j}^{\alpha}} x \in \overline{\operatorname{co}}\left\{T_{j, t} x: t \in G\right\}$ for all $\alpha \in I$ and $\overline{\operatorname{co}}\left\{T_{j, t} x: t \in G\right\}$ is weakly compact, there exists a subnet $\left\{T_{j, \mu_{j}^{\beta}}^{\beta} x: \beta \in J\right\}$ of $\left\{T_{j, \mu_{j}^{\alpha}} x: \alpha \in I\right\}$ such that $T_{j, \mu_{j}^{\beta}} x$ converges weakly to an element $x_{0} \in \overline{c o}\left\{T_{j, t} x: t \in G\right\}$. To show that $T_{j, \mu_{j}^{\alpha}} x$ converges weakly to $Q_{j} x$, it is sufficient to show $x_{0}=Q_{j} x$. Let $x^{*} \in E^{*}$ and $s \in G$. Since $T_{j, \mu_{j}^{\beta}} x \rightarrow x_{0}$ weakly, we have $\mu_{j, t}^{\beta}\left\langle T_{j, t} x, x^{*}\right\rangle \rightarrow\left\langle x_{0}, x^{*}\right\rangle$ and $\mu_{j, t}^{\beta}\left\langle T_{j, t} x, T_{j, s}^{*} x^{*}\right\rangle \rightarrow\left\langle x_{0}, T_{j, s}^{*} x^{*}\right\rangle=\left\langle T_{j, s} x_{0}, x^{*}\right\rangle$. On the other hand, since $\mu_{j}^{\beta}-l_{s}^{*} \mu_{j}^{\beta} \rightarrow 0$ in the weak* topology, we have

$$
\begin{aligned}
& \mu_{j, t}^{\beta}\left\langle T_{j, t} x, x^{*}\right\rangle-l_{s}^{*} \mu_{j, t}^{\beta}\left\langle T_{j, t} x, x^{*}\right\rangle \\
& =\mu_{j, t}^{\beta}\left\langle T_{j, t} x, x^{*}\right\rangle-\mu_{j, t}^{\beta}\left\langle T_{j, s t} x, x^{*}\right\rangle \\
& \quad=\mu_{j, t}^{\beta}\left\langle T_{j, t} x, x^{*}\right\rangle-\mu_{j, t}^{\beta}\left\langle T_{j, t} x, T_{j, s}^{*} x^{*}\right\rangle \\
& \quad \rightarrow 0 .
\end{aligned}
$$

Hence, we have $\left\langle x_{0}, x^{*}\right\rangle=\left\langle T_{j, s} x_{0}, x^{*}\right\rangle$ and hence $x_{0} \in F_{j}(G)$. So, we obtain $Q_{j} x=T_{j, \mu_{j}} x=x_{0}$ from Lemma 3.4. That the projection $Q_{j}$ is the same for all asymptotically invariant nets is obvious from Lemma 3.3.

As direct consequence of Theorem 4.1, we have the following.

Corollary 4.2 Let $\left\{T_{j, t}: t \in G\right\}$ be as in Theorem 4.1 and assume that an invariant mean exists on $G$. Then there exists a projection $Q_{j}$ of $E$ onto $F_{j}$ such that $\left\|\left(Q_{1}, \ldots, Q_{j}\right)\right\|_{j} \leq C$, $Q_{j} T_{j, t}=T_{j, t} Q_{j}=Q_{j}$ for each $t \in G$ and $Q_{j} x \in \overline{\mathrm{co}}\left\{T_{j, t} x: t \in G\right\}$ for each $x \in E$.

Theorem 4.3 Let $\left\{T_{j, t}: t \in G\right\}$ be as in Theorem 4.1. If a net $\left\{\mu_{j}^{\alpha}: \alpha \in I\right\}$ of means on $G$ is asymptotically invariant and further $\mu_{j}^{\alpha}-r_{s}^{*} \mu_{j}^{\alpha}$ converges to 0 in the strong topology of $m_{j}(G)^{*}$, then there exists a projection $Q_{j}$ of $E$ onto $F_{j}(G)$ such that $\left\|\left(Q_{1}, \ldots, Q_{j}\right)\right\|_{j} \leq C$, $T_{j, \mu_{j}^{\alpha}} x$ converges strongly to $Q_{j} x$ for each $x \in E, Q_{j} T_{j, t}=T_{j, t} Q_{j}=Q_{j}$ for each $t \in G$, and $Q_{j} x \in$ $\overline{\mathrm{co}}\left\{T_{j, t} x: t \in G\right\}$ for each $x \in E$.

Proof As in the proof of Theorem 4.1, let $Q_{j}=T_{j, \mu_{j}}$, where $\mu_{j}$ is a cluster point of the net $\left\{\mu_{j}^{\alpha}: \alpha \in I\right\}$ in the weak ${ }^{*}$ topology of $m_{j}(G)^{*}$. We show that $T_{j, \mu_{j}^{\alpha}} x$ converges strongly to $Q_{j} x$ for each $x \in E$.

Let $E_{0}=\overline{\operatorname{co}}\left\{y-T_{j, t} y: y \in E, t \in G\right\}$. Then, for any $z \in E_{0}, T_{j, \mu_{j}^{\alpha}} z$ converges strongly to 0 . In fact, if $z=y-T_{j, s} y$, then since, for any $y^{*} \in E^{*}$,

$$
\begin{aligned}
\left|\left\langle T_{j, \mu_{j}^{\alpha}} z, y^{*}\right\rangle\right| & \left.=\left|\mu_{j, t}^{\alpha}\right| T_{j, t}\left(y-T_{j, s} y\right), y^{*}\right\rangle \mid \\
& \left.=\left|\mu_{j, t}^{\alpha}\right| T_{j, t} y, y^{*}\right\rangle-\mu_{j, t}^{\alpha}\left\langle T_{j, t s} y, y^{*}\right\rangle \mid \\
& =\left|\left(\mu_{j, t}^{\alpha}-r_{s}^{*} \mu_{j, t}^{\alpha}\right)\left\langle T_{j, t} y, y^{*}\right\rangle\right|
\end{aligned}
$$




$$
\begin{aligned}
& \leq\left\|\left(\mu_{1}^{\alpha}-r_{s}^{*} \mu_{1}^{\alpha}, \ldots, \mu_{j}^{\alpha}-r_{s}^{*} \mu_{j}^{\alpha}\right)\right\|_{j} \cdot \sup _{t}\left|\left\langle T_{j, t} y, y^{*}\right\rangle\right| \\
& \leq\left\|\left(\mu_{1}^{\alpha}-r_{s}^{*} \mu_{1}^{\alpha}, \ldots, \mu_{j}^{\alpha}-r_{s}^{*} \mu_{j}^{\alpha}\right)\right\|_{j} \cdot C \cdot\|y\|_{j} \cdot\left\|y^{*}\right\|_{j},
\end{aligned}
$$

we have $\left\|\left(T_{1, \mu_{1}^{\alpha}} z, \ldots, T_{j, \mu_{j}^{\alpha}} z\right)\right\|_{j} \leq C \cdot\left\|\left(\mu_{1}^{\alpha}-r_{s}^{*} \mu_{1}^{\alpha}, \ldots, \mu_{j}^{\alpha}-r_{s}^{*} \mu_{j}^{\alpha}\right)\right\|_{j} \cdot\|y\|_{j}$. Using this inequality, we show that $T_{j, \mu_{j}^{\alpha}} z$ converges strongly to 0 for any $z \in E_{0}$. Let $z$ be any element of $E_{0}$ and $\epsilon$ be any positive number. By the definition of $E_{0}$, there exists an element $\sum_{i=1}^{n} a_{i}\left(y_{i}-\right.$ $\left.T_{j, s_{i}} y_{i}\right) \epsilon$ in the set $\operatorname{co}\left\{y-T_{j, s} y: y \in E, s \in G\right\}$ such that $\epsilon>2 C \cdot \|\left(z-\sum_{i=1}^{n} a_{i}\left(y_{i}-T_{1, s_{i}} y_{i}\right), \ldots, z-\right.$ $\left.\sum_{i=1}^{n} a_{i}\left(y_{i}-T_{j, s_{i}} y_{i}\right)\right) \|_{j}$. On the other hand, from $\left\|\left(\mu_{1}^{\alpha}-r_{s}^{*} \mu_{1}^{\alpha}, \ldots, \mu_{j}^{\alpha}-r_{s}^{*} \mu_{j}^{\alpha}\right)\right\|_{j} \rightarrow 0$ for all $s \in G$, there exists $a_{0} \in I$ such that, for all $\alpha \geq \alpha_{0}$ and $i=1,2, \ldots, n$,

$$
\epsilon>\left\|\left(\mu_{1}^{\alpha}-r_{s_{i}}^{*} \mu_{1}^{\alpha}, \ldots, \mu_{j}^{\alpha}-r_{s_{i}}^{*} \mu_{j}^{\alpha}\right)\right\|_{j} \cdot 2 C\left\|y_{i}\right\|_{j} .
$$

This yields

$$
\begin{aligned}
& \left\|\left(T_{1, \mu_{1}^{\alpha}} z, \ldots, T_{j, \mu_{j}^{\alpha}} z\right)\right\|_{j} \\
& \leq \|\left(T_{1, \mu_{1}^{\alpha}} z-T_{1, \mu_{1}^{\alpha}}\left(\sum_{i=1}^{n} a_{i}\left(y_{i}-T_{1, s_{i}} y_{i}\right)\right),\right. \\
& \left.\ldots, T_{j, \mu_{j}^{\alpha}} z-T_{j, \mu_{j}^{\alpha}}\left(\sum_{i=1}^{n} a_{i}\left(y_{i}-T_{j, s_{i}} y_{i}\right)\right)\right) \|_{j} \\
& +\left\|\left(T_{1, \mu_{1}^{\alpha}}\left(\sum_{i=1}^{n} a_{i}\left(y_{i}-T_{1, s_{i}} y_{i}\right)\right), \ldots, T_{j, \mu_{j}^{\alpha}}\left(\sum_{i=1}^{n} a_{i}\left(y_{i}-T_{j, s_{i}} y_{i}\right)\right)\right)\right\|_{j} \\
& \leq\left\|\left(T_{1, \mu_{1}^{\alpha}}, \ldots, T_{j, \mu_{j}^{\alpha}}^{\alpha}\right)\right\|_{j} \\
& \cdot\left\|\left(z-\sum_{i=1}^{n} a_{i}\left(y_{i}-T_{1, s_{i}} y_{i}\right), \ldots, z-\sum_{i=1}^{n} a_{i}\left(y_{i}-T_{j, s_{i}} y_{i}\right)\right)\right\|_{j} \\
& +\sum_{i=1}^{n}\left\|\left(T_{1, \mu_{j}^{\alpha}}\left(y_{i}-T_{1, s_{i}} y_{i}\right), \ldots, T_{j, \mu_{j}^{\alpha}}\left(y_{i}-T_{j, s_{i}} y_{i}\right)\right)\right\|_{j} \\
& \leq C \cdot\left\|\left(z-\sum_{i=1}^{n} a_{i}\left(y_{i}-T_{1, s_{i}} y_{i}\right), \ldots, z-\sum_{i=1}^{n} a_{i}\left(y_{i}-T_{j, s_{i}} y_{i}\right)\right)\right\|_{j} \\
& +\sup _{i}\left\|\left(\mu_{1}^{\alpha}-r_{s_{i}}^{*} \mu_{1}^{\alpha}, \ldots, \mu_{j}^{\alpha}-r_{s_{i}}^{*} \mu_{j}^{\alpha}\right)\right\|_{j} \cdot C \cdot\left\|y_{i}\right\|_{j} \\
& <\frac{\epsilon}{2}+\frac{\epsilon}{2}=\epsilon \text {. }
\end{aligned}
$$

Hence, $T_{j, \mu_{j}^{\alpha}} Z$ converges strongly to 0 for each $z \in E_{0}$.

Next, assume that $x-T_{j, \mu_{j}} x$ for some $x \in E$ is not contained in the set $E_{0}$. Then, by the Hahn-Banach theorem, there exists a linear continuous functional $y^{*}$ such that $\langle x-$ $\left.T_{j, \mu_{j}} x, y^{*}\right\rangle=1$ and $\left\langle z, y^{*}\right\rangle=0$ for all $z \in E_{0}$. So since $x-T_{j, t} x \in E_{0}$ for all $t \in G$, we have

$$
\left\langle x-T_{j, \mu_{j}} x, y^{*}\right\rangle=\mu_{j, t}\left\langle x-T_{j, t} x, y^{*}\right\rangle=0 .
$$

This is a contradiction. Hence, $x-T_{j, \mu_{j}}$ for all $x \in E$ are contained in $E_{0}$. Therefore we find that $T_{j, \mu_{j}^{\alpha}} x-T_{j, \mu_{j}} x=T_{j, \mu_{j}^{\alpha}}\left(x-T_{j, \mu_{j}}\right)$ converges strongly to 0 for all $x \in E$. This completes the proof. 
By using Theorem 4.3, we can obtain the following corollary.

Corollary 4.4 Let $E$ be a real Banach space and $T_{j}$ be a linear operator of $E$ into itself such that exists a constant $C$ with $\left\|\left(T_{1}^{n}, \ldots, T_{j}^{n}\right)\right\|_{j} \leq C$ for $n=1,2, \ldots$, and $T_{j}$ is weakly completely continuous, i.e., $T_{j}$ maps the closed unit ball of $E$ into a weakly compact subset of $E$. Then there exists a projection $Q_{j}$ of $E$ onto the set $F_{j}(T)$ of all fixed point of $T_{j}$ such that $\left\|\left(Q_{1}, \ldots, Q_{j}\right)\right\|_{j} \leq C$, the Cesaro means $S_{j, n}=\frac{1}{n} \sum_{k=1}^{n} T_{j}^{k} x$ converges strongly to $Q_{j} x$ for each $x \in E$, and $T_{j} Q_{j}=Q_{j} T_{j}=Q_{j}$.

Proof Let $x \in E$. Then, since $\left\{T_{j}^{n} x: n=1,2, \ldots\right\}=T_{j}\left(\left\{T^{n-1} x: n=1,2, \ldots\right\}\right) \subset T_{j}(B(0,\|x\| \cdot$ $(c+1)))$, where $B(x, r)$ means the closed ball with center $x$ and radius $r$, the weak closure of $\left\{T_{j}^{n} x: n=1,2, \ldots\right\}$ is weakly compact. On the other hand, let $G=\{1,2,3, \ldots\}$ with the discrete topology and $\mu_{j}^{n}$ be a mean on $G$ such that $\mu_{j}^{n}\left(f_{j}\right)=\sum_{i=1}^{n}\left(\frac{1}{n}\right) f_{j}(i)$ for each $f_{j} \in m_{j}(G)$. Then it is obvious that $\left\|\left(\mu_{1}^{n}-r_{k}^{*} \mu_{1}^{n}, \ldots, \mu_{j}^{n}-r_{k}^{*} \mu_{j}^{n}\right)\right\|_{j} \leq \frac{2 k}{n} \rightarrow 0$ for all $k \in G$. So, it follows from Theorem 4.3 that Corollary 4.4 is true.

If $G=[0, \infty)$ with the natural topology, then we obtain the corresponding result.

Corollary 4.5 Let $E$ be a real Banach space and $\left\{T_{j, t}: t \in[0, \infty)\right\}$ be a family of linear operators of $E$ into itself satisfying Assumption (A). Then there exists a projection $Q_{j}$ of $E$ onto $F_{j}(G)$ such that $\left\|\left(Q_{1}, \ldots, Q_{j}\right)\right\|_{j} \leq C, \frac{1}{T} \int_{0}^{T} T \int_{j, t} x d t$ converges strongly to $Q_{j} x$ for each $x \in E$, and $T_{j, t} Q_{j}=Q_{j} T_{j, t}=Q_{j}$ for each $t \in[0, \infty)$.

Remark 4.6 $\frac{1}{T} \int_{0}^{T} T \int_{j, t} x d t$ are weak vector valued integrals with respect to means on $G=[0, \infty)$. As in Section IV of Rodé [4], we can also obtain the strong convergence of the sequences

$$
(1-r) \sum_{k=1}^{\infty} r^{k} T_{j}^{k} x, \quad r \rightarrow 1-
$$

and

$$
\lambda \int_{0}^{\infty} e^{-\lambda t} T_{j, t} x d t, \quad \lambda \rightarrow 0+
$$

\section{Competing interests}

The authors declare that they have no competing interests.

\section{Authors' contributions}

All authors carried out the proof. All authors conceived of the study, and participated in its design and coordination. All authors read and approved the final manuscript.

\section{Author details}

${ }^{1}$ Department of Mathematics, Science and Research Branch, Islamic Azad University, Ashrafi Esfahani Ave., Tehran, 14778, Iran. ${ }^{2}$ Department of Mathematics and Computer Science, Iran University of Science and Technology, Tehran, Iran.

${ }^{3}$ Department of Mathematics Education and the RINS, Gyeongsang National University, Jinju, 660-701, Korea.

\section{Acknowledgements}

Yeol Je Cho was supported by the Basic Science Research Program through the National Research Foundation of Korea (NRF) funded by the Ministry of Education, Science and Technology (Grant Number: 2013053358). 


\section{References}

1. Yosida, K: Mean ergodic theorem in Banach spaces. Proc. Imp. Acad. (Tokyo) 14(8), 292-294 (1938)

2. Baillon, J-B: Un théorème de type ergodique pour les contractions non linéaires dans un espace de Hilbert. C. R. Acad. Sci. Paris, Sér. A-B 280, A1511-A1514 (1975) (French)

3. Pazy, A: On the asymptotic behavior of iterates of nonexpansive mappings in Hilbert space. Isr. J. Math. 26(2), 197-204 (1977)

4. Rodé, G: An ergodic theorem for semigroups of nonexpansive mappings in a Hilbert space. J. Math. Anal. Appl. 85(1), $172-178(1982)$

5. Takahashi, W: A nonlinear ergodic theorem for an amenable semigroup of nonexpansive mappings in a Hilbert space. Proc. Am. Math. Soc. 81(2), 253-256 (1981)

6. Greenleaf, FP: Invariant Means on Topological Groups and Their Applications. Van Nostrand Mathematical Studies, vol. 16. Van Nostrand-Reinhold, New York (1969)

7. Takahashi, W: Invariant functions for amenable semigroups of positive contractions on $L^{1}$. Kodai Math. Semin. Rep. 23, 131-143 (1971)

8. Hirano, N, Takahashi, W: Nonlinear ergodic theorems for an amenable semigroup of nonexpansive mappings in a Banach space. Pac. J. Math. 112(2), 333-346 (1984)

9. Kido, K, Takahashi, W: Mean ergodic theorems for semigroups of linear operators. J. Math. Anal. Appl. 103(2), 387-394 (1984)

10. Dales, HG, Polyakov, ME: Multi-normed spaces. Diss. Math. 488, 1-165 (2012)

11. Dales, HG, Moslehian, MS: Stability of mappings on multi-normed spaces. Glasg. Math. J. 49(2), $321-332$ (2007)

12. Moslehian, MS, Nikodem, K, Popa, D: Asymptotic aspect of the quadratic functional equation in multi-normed spaces. J. Math. Anal. Appl. 355(2), 717-724 (2009)

13. Park, C, Saadati, R: Approximation of a generalized additive mapping in multi-Banach modules and isomorphisms in multi-C*-algebras: a fixed-point approach. Adv. Differ. Equ. 2012, 162 (2012)

14. O'Regan, D, Rassias, JM, Saadati, R: Approximations of ternary Jordan homomorphisms and derivations in multi-C ternary algebras. Acta Math. Hung. 134(1-2), 99-114 (2012)

15. Agarwal, RP, Cho, YJ, Park, C, Saadati, R: Approximate homomorphisms and derivation in multi-Banach algebras. Comment. Math. 51(1), 23-38 (2011)

16. Kenari, HM, Saadati, R, Cho, YJ: The mean ergodic theorem for nonexpansive mappings in multi-Banach spaces. J. Inequal. Appl. 2014, 259 (2014)

17. Day, MM: Amenable semigroups. III. J. Math. 1, 509-544 (1957)

18. Saluja, GS: Weak convergence theorems for two asymptotically quasi-nonexpansive non-self mappings in uniformly convex Banach spaces. J. Nonlinear Sci. Appl. 7(2), 138-149 (2014)

19. Thianwan, T: Convergence criteria of modified Noor iterations with errors for three asymptotically nonexpansive nonself-mappings. J. Nonlinear Sci. Appl. 6(3), 181-197 (2013)

20. Purtas, Y, Kiziltunc, H: Weak and strong convergence of an explicit iteration process for an asymptotically quasi-i-nonexpansive mapping in Banach spaces. J. Nonlinear Sci. Appl. 5(5), special issue, $403-411$ (2012)

10.1186/1029-242X-2014-402

Cite this article as: Kenari et al.: Mean ergodic theorem for semigroups of linear operators in multi-Banach spaces. Journal of Inequalities and Applications 2014, 2014:402

\section{Submit your manuscript to a SpringerOpen ${ }^{\circ}$ journal and benefit from:}

- Convenient online submission

- Rigorous peer review

Immediate publication on acceptance

- Open access: articles freely available online

- High visibility within the field

- Retaining the copyright to your article 